\title{
UNUSUAL MANIFESTATIONS ÖF PULMONARY EMBOLIC DISEASE
}

\author{
M. Hamilton, M.D., M.R.C.P. \\ Physician, \\ E. N. Thompson, M.B., M.R.C.P.* \\ Registrar,
}

Chelmsford Group of Hospitals

Pulmonary embolic disease is now well recognized as presenting two main clinical stateseither a pneumonia-like illness, following a solitary pulmonary embolus, or cardiac failure due to widespread obliteration of the pulmonary vascular tree, with many small emboli.

We here describe three further unusual methods of presentation of this common condition: (a) Resembling bacterial endocarditis, (b) simulating gastro-intestinal disease, and $(c)$ causing transient cerebral ischæmia.

\section{Pulmonary Embolic Disease Resembling Bacterial Endocarditis \\ Case I}

A. T., a 68-year-old housewife, was admitted to hospital on 29.1.60. She had been well until one month previously, when she had been woken from sleep by a severe substernal constricting pain, associated with dyspnœa, which persisted for several hours, until finally relieved by a morphine injection. Although experiencing no pain subsequently, she remained breathless on slight exertion. On admission to hospital, she was in gross cardiac failure, with signs of combined ventricular hypertrophy, with a harsh ejection systolic, and soft early diastolic murmur, heard down the left sternal edge. The heart was in sinus rhythm and the B.P. $150 / 80 \mathrm{~mm}$. Hg. Both liver and spleen were enlarged $\mathrm{I} \frac{1}{2}$ and $\frac{1}{2}$ in. respectively below the costal margin. She was afebrile, with no digital clubbing, the urine contained albumin, occasional hyaline and granular casts and red blood cells; both the white blood cell count and erythrocyte sedimentation rate were normal.

Despite conventional therapy, the heart failure progressed and the deterioration was associated with the development of pyrexia up to $10 I^{\circ} \mathbf{F}$, which persisted for the next two months (Fig. I), accompanied by a polymorph-leucocytosis of $12,000 / \mathrm{cu} . \mathrm{mm}$., and a rise in the sedimentation rate to $30 \mathrm{~mm}$. $/ \mathrm{hr}$. Although numerous blood cultures proved sterile, a diagnosis of subacute bacterial endocarditis affecting the aortic valve was made, and antibiotic treatment started. However, this did not effect any improvement in her condition, the heart failure slowly progressed, and the patient died five months after admission to hospital. Post-mortem examination showed many emboli, of varying ages, blocking most of the small branches of the pulmonary arteries throughout both lungs. The right ventricle was hypertrophied and the right auricle dilated, with stretched tricuspid valve ring, but no other valve abnor-

* Present address: Department of Medicine, Royal Free Hospital. mality, and no changes to suggest that the patient had ever had bacterial endocarditis. There was extensiven thrombosis in the veins of both calves.

\section{Case 2}

A 66-year-old housewife, was admitted to hospital in gross cardiac failure on 29. I 1.6I. Three weeks pre- $A$ viously, whilst resting, she had experienced an episode of intense dyspnœa, lasting for one hour, and this had been succeeded by increasing effort dyspnoea and orthop $+\vec{t}$ nœa and a succession of small hæmoptyses. On the day ofo admission she had experienced a typical right-sidech pleuritic pain. On examination she was in gross cardiacfailure with rapid auricular fibrillation. The blood pressure was $130 / 90 \mathrm{~mm}$. $\mathrm{Hg}$. There was a basal systoli murmur with clinical and ECG evidence of left ven $\triangle$ tricular hypertrophy. She was febrile with a temperature of $101^{\circ} \mathrm{F}$. and had a polymorph-leucocytosis of $I I, 0 \%$ cu. mm., but the ESR was not raised. The urine con tained albumin with red and white blood cells; सुझల initial blood urea was $74 \mathrm{mg}$./100 $\mathrm{ml}$. Despite the 3 운 sence of digital clubbing, splenomegaly, Osler's nodes. and persistently negative blood cultures, a diagnosis of aortic bacterial endocarditis was made and a rise in the blood urea to $350 \mathrm{mg}$. $/ 100 \mathrm{ml}$. was considered strongD supporting evidence. Although conventional antibiotic therapy, combined with treatment for the cardiac failure $\overline{\bar{D}}$ was started, the spleen became palpable, an aortic diastolic murmur developed, the fever and cardiac failure persisted; the blood urea fell to under $100 \mathrm{mg} . / 100 \mathrm{ml}$ as a result of rigid dietary regime. Three weeks after admission evidence of venous thrombosis in the leg became obvious and was soon followed by intermittent hæmoptysis, increasing cardiac failure and increased pyrexia. At this stage a diagnosis of pulmonary embolie disease was considered most likely, particularly as the response to adequate antibiotic therapy had not been successful. Anticoagulant therapy was commenced with subsidence of fever and cardiac failure and with ne further progression of the symptoms or signs. On\& month later the patient was discharged home and is nowo (six months later) fully active in her household duties.

\section{Summary}

Both patients presented in gross cardiac failure with signs suggestive of valvular disease. In view of the fever, leucocytosis, changing cardiac murs murs and increasing heart failure the diagnosis of bacterial endocarditis seemed probable. Case at no time showed any evidence of venous throm: bosis or pulmonary embolic disease either clinic $-\overline{-}$ ally, radiologically or cardiographically. The second patient had features of pulmonary emboliळ̄ 


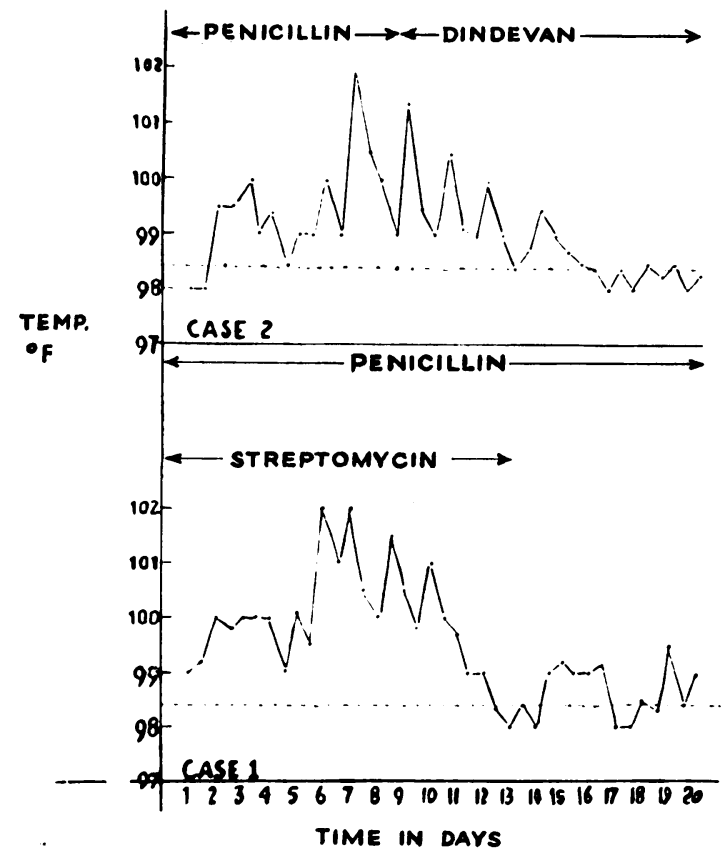

FIG. I.-Illustraring the prolonged and persistent fever associated with pulmonary embolic disease.

disease initially, but in view of the history of rheumatic heart disease and evidence of aortic valve involvement the diagnosis of bacterial endocarditis seemed more probable. However, there was no response to antibiotic treatment, and only when anticoagulant therapy was given did improvement occur.

\section{Resembling Gastro-intestinal Disease}

\section{Case 3}

A 75-year-old male patient was admitted to hospital with a ten weeks' history of vague upper abdominal pain and occasional vomitus of clear fluid. For several months he had complained of increasing effort intolerance and orthopnoea and one month previously had developed a cough and recurrent hæmoptysis. On admission he was in gross cardiac failure with rapid auricular fibrillation and evidence of combined ventricular hypertrophy. The blood pressure was $170 / 100 \mathrm{~mm}$. Hg. He was obviously jaundiced with abdominal distension due to a firm irregular enlargement of the liver 4 in. below the costal margin and ascites. Rectal examination was negative. In view of the abdominal pain, vomiting, hepatomegaly, ascites and jaundice, he was admitted to hospital thought to be suffering from a gastro-intestinal neoplasm with hepatic metastases. However, he was also in gross cardiac failure with a history suggestive of pulmonary embolic disease, particularly as there was also evidence of venous thrombosis in the right calf veins at the time of admission. The chest X-ray showed an area of consolidation at the right base with elevation of the diaphragm and enlargement of both hilar shadows (Fig. 2). The ECG showed only auricular fibrillation. The serum bilirubin was I. $6 \mathrm{mg}$./ $100 \mathrm{ml}$., other liver function tests being normal, as was the white blood cell count. During the next

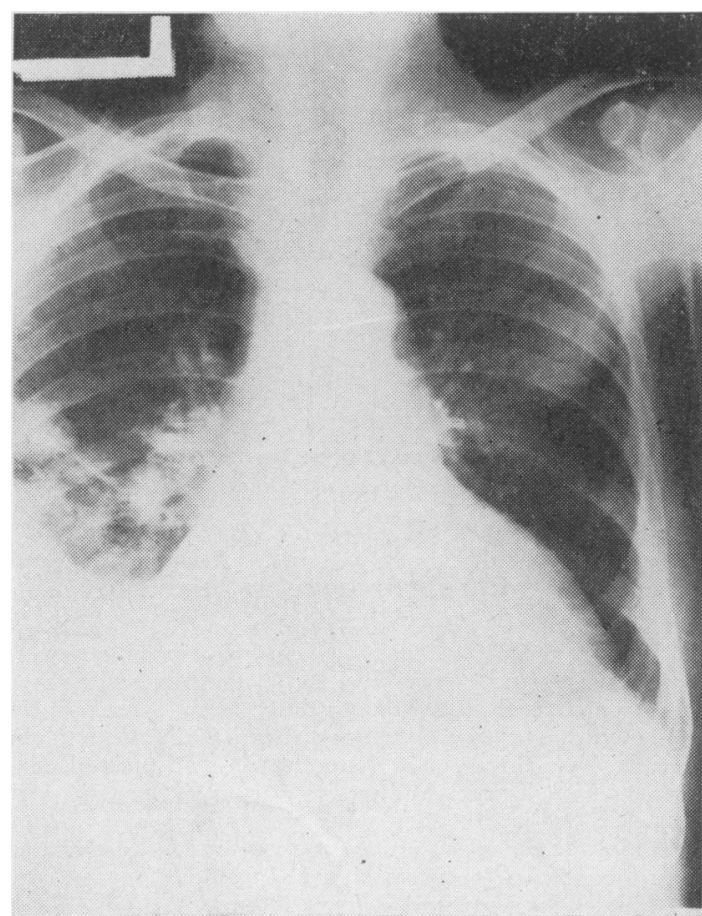

FIG. 2.-Chest X-ray (Case 3) showing peripheral consolidation with pleural effusion.

month the cardiac failure subsided considerably with therapy, but the jaundice persisted. However, chest $\mathrm{X}$-ray showed increased consolidation at the right base and enlargement of the right hilar shadow, suggesting that he had a bronchogenic carcinoma with hepatic secondaries. Despite deteriorating radiological appearances, the patient improved symptomatically until two months after admission, when he suddenly became intensely dyspnoic and died within a few minutes. Autopsy showed the presence of many pulmonary infarcts and organizing thrombus in many of the pulmonary arteries in both lungs. There was evidence of bilateral venous thrombosis in both calf veins.

\section{Case 4}

A 7I-year-old male with a two-year history of chronic cardiac failure, auricular fibrillation, adequately treated with digitalis and diuretics, and thus able to maintain a fairly active life. Three days before admission he became jaundiced and passed three large melæna stools. This was followed by increased dyspnœa at rest and also orthopnœa. On examination he was markedly jaundiced and in gross cardiac failure. He was drowsy and unable to give an adequate history. The rhythm was irregular due to auricular fibrillation and there was evidence of left ventricular hypertrophy. The blood pressure was unobtainable. Râles were present at both lung bases. The liver was slightly enlarged and there was fresh melæna stool on rectal examination. Other systems, including examination of legs, was normal.

In view of the jaundice, hepatomegaly and melæna, it was thought that he had a gastric neoplasm with hepatic deposits, in addition to cardiac failure. His condition deteriorated rapidly and he died 18 hours after admission. Autopsy showed no gastro-intestinal abnormality. The upper air passages contained copious 
quantities of fresh blood, much of which had been swallowed. There was a massive recent pulmonary embolus wedged in the main pulmonary artery, also evidence of other smaller emboli packing the arteries of both lungs which showed extensive hæmorrhagic infarction. There was extensive thrombosis of deep veins of both legs. All other systems were normal, but the intestine was filled with swallowed blood.

\section{Summary}

Both patients, in gross cardiac failure, presented with jaundice, ascites and hepatomegaly-in Case 3 the diagnosis of pulmonary embolic disease was made with confidence, but the profuse melæna occurring in Case 4 was taken to indicate a primary gastro-intestinal lesion so that the correct diagnosis was not considered.

\section{Resembling Cerebro-vascular Disease Case 5}

A 65-year-old female with a two-year history of hypertension and congestive cardiac failure which had been treated with digitalis and diuretics. Three months before admission she developed thrombophlebitis in the left calf, associated with 'bronchitis', and treated conservatively at home. She was admitted to hospital with a week's history of increasing drowsiness and mental confusion with periods of loss of consciousness associated with incontinence, followed by a period of vague and irrational behaviour and slurred speech. No convulsive movements were observed during these attacks. She was febrile with a temperature of $101^{\circ} \mathrm{F}$. and was confused, disorientated and uncooperative, with slurred speech There were no localizing signs on neurological examination and no meningism: the fundi were normal except for vessel changes. There was evidence of left ventricular hypertrophy with signs of cardiac failure with a raised venous pressure, basal râles and peripheral œdema; the pulse was regular and the blood pressure $180 / 120$ $\mathrm{mm}$. $\mathrm{Hg}$. In view of the rapidly progressive mental deterioration, associated with episodes of unconsciousness, a diagnosis of a cerebral tumour seemed most likely. Investigations performed were unhelpful in elucidating the diagnosis. Her condition deteriorated rapidly; she lapsed into unconsciousness and died four days after admission. Autopsy showed a massive recent pulmonary embolus together with smaller, older pulmonary emboli packing the smaller arteries in both lungs. There was extensive thrombosis in veins of both calves. The brain appeared normal.

Despite the previous history of ' bronchitis' associated with thrombophlebitis in the legs, this present illness simulated a primary cerebral condition. There was no clinical or obvious radiological evidence of pulmonary embolic disease or active thrombophlebitis.

\section{Case 6}

A 67-year-old male had been previously fit and ambulant, apart from three weeks' history of 'odd behaviour' which had been noted by his neighbour, who had found him unconscious on the day of admission to hospital. On examination he was conscious but confused and disorientated and aphasic. There was no soft tissue injury of the scalp and no meningism; the fundi were normal except for vessel changes and there were signs of right hemiparesis. $\mathrm{He}$ was in cardiac. failure with a raised venous pressure, peripheral œdema and râles at the lung bases with slight left ventricular enlargement of the heart, which was in regular rhythm; the blood pressure was $170 / 95 \mathrm{~mm}$. Hg. Examination of other systems was normal. Soon after admission he $\overrightarrow{0}$ had several generalized convulsions, but no change in the physical signs except for deteriorating level of con- 은 sciousness. Lumbar puncture yielded normal CSF at normal pressure. The blood count was normal, the ESR Q $23 \mathrm{~mm}$./hr. and serum electrolytes normal. The chest $\subseteq$

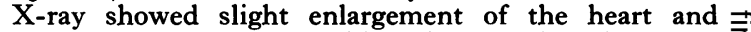
increased pulmonary and hilar shadows, but the X-ray $\vec{\omega}$ of the skull was normal. The fits were controlled with $\overline{0}$ phenobarbitone and the congestive failure treated. Two days after admission he was fully conscious, with normal $\overline{\bar{\omega}}$ speech and no evidence of hemiparesis. Eight days later $\frac{\omega}{D}$ he had a further generalized convulsion and rapidly $\mathbb{Q}$ succumbed. Autopsy showed a massive fresh pulmonary embolus in both main pulmonary arteries together with $\omega$ packing of smaller vessels with older emboli.

The heart showed hypertrophy of both ventricles. The brain showed evidence of a small old infarct in the $\overrightarrow{\vec{\omega}}$ right cerebellar hemisphere, the rest of the brain $\omega$ appeared normal. There was extensive thrombo- $\frac{D}{0}$ phlebitis in the deep veins of both legs.

\section{Case 7}

A 60-year-old diabetic previously well controlled on oral therapy until the morning of admission, when she of had suddenly lost consciousness and had a generalized $N$ convulsion. Her previous health had been good, there $\stackrel{\infty}{\infty}$ being no history of fits or faints.

On examination she was in status epilepticus with 을 deviation of eyes to the right. The tendon reflexes were increased on the right side with bilateral extensor $c$ plantar responses. There was no meningism and the $\subseteq$ fundi were normal. She was febrile with a temperature $\overparen{D}$ fluctuating between 99 and $102^{\circ} \mathrm{F}$. There were signs $\vec{\oplus}$ of cardiac failure, slight enlargement of the hearo which was in regular rhythm; the blood pressure wa $\omega$ $200 / 100 \mathrm{~mm}$. Hg. Examination of other systems w. normal.

Lumbar puncture showed clear fluid, under normat pressure, with no cellular or chemical abnormality. The $\bar{D}$ blood count and ESR were normal, as were the serum electrolytes and urine. The blood sugar on admission $\mathbb{D}$ was $540 \mathrm{mg} . / 100 \mathrm{ml}$. The fits were controlled with phenobarbitone and the diabetes stabilized on insulin. $\bar{\partial}$ The day after admission she was conscious with signs of 3 right-sided hemiparesis and slurred speech. There was a $\supset$ steady improvement and by the fourth day there were no abnormal physical signs. She remained well until the tenth day, when there was a recurrence of the convulsions; she died half an hour later. Autopsy showed a massive recent pulmonary embolus together with evidence of older emboli obliterating the smaller arteries in both lungs. There was enlargement of the heart affecting both ventricles. The brain appeared small, $\frac{0}{3}$ owing to generalized senile atrophy, but there was no hæmorrhage or infarct. There was extensive phlebothrombosis in veins of both calves.

\section{Summary}

All three elderly patients presented with an $\stackrel{N}{\Omega}$ illness characterized by neurological features, viz. N coma, fits and transient pyramidal signs. Although $\mathrm{N}$ all had undoubted cerebral vascular disease, the event which precipitated the final neurological 0 illness was circulatory.

\section{Discussion}

Despite the varying clinical impressions of these patients, several features have been common to all.

\section{.}


Fever was marked in all cases, the temperature ranging between 100 and $103^{\circ} \mathrm{F}$. maintained over a prolonged period and associated with a slight polymorph leucocytosis of 10,000 to $12,000 /$ cu.mm. and raised ESR to 20 to $30 \mathrm{~mm}$. $/ \mathrm{hr}$. Despite the prolonged fever there was a definite lack of inanition; these patients never looked as ill as would be expected if the fever had had an infective basis. Also the complete lack of response to antibiotic therapy was suggestive evidence that the fever was not due to an infection. This fever was particularly pronounced in Cases I and 2 and was one of the chief reasons which led to an error in diagnosis (Fig. I).

Cardiac failure was a feature in all cases, and the poor response to conventional therapy was marked. Right ventricular preponderance was not obvious, but rather a combined ventricular hypertrophy with slight accentuation of the main pulmonary vessels radiologically. Radiological confirmation of pulmonary emboli was lacking except in Case 3 and then the signs were localized to the right lower lobe and right hilar region. All these patients, whatever the mode of presentation, showed the above two features. The mechanism of heart failure resulting from recurrent pulmonary emboli has already been described (Owen, Thomas, Castelman and Bland, 1953). However, this important feature was overshadowed by other manifestations described.

Had we paid sufficient regard to the cardiac failure the correct diagnosis would not have proved so elusive. All had been in heart failure before the onset of their final illness and during this time had presumably been lodging emboli in the small pulmonary arteries. The sudden advent of new features associated with a rapid deterioration in the patient's condition did not indicate another disease, but merely an increased severity of that which had been in existence for some time. It seems probable that, had the initial pulmonary embolic disease been more rapid, the predominant feature would have been cardiac failure, rather than other system involvement. If more notice had been taken of the unexplained cardiac failure the diagnosis of recurrent pulmonary emboli should have been considered during life. All these patients were elderly and had associated ischæmic heart disease, and it was assumed that the failure was due to this, rather than obliterative cor pulmonale. Fever, with a cardiac lesion causing progressive cardiac failure, particularly if this is also associated with a change in character of the cardiac murmur, or the actual development of such murmurs, naturally raises the possibility that the patient suffers from bacterial endocarditis. That such murmurs do develop during the course of pulmonary embolic disease has previously been recognized and attributed to turbulence in a partially occluded pulmonary artery (Owen and others). Clubbing of the fingers, which may develop during the course of pulmonary embolic disease (Thompson and Hamilton, 1962), adds greater strength to this diagnosis, even if no other signs associated with bacterial endocarditis develop. Case I also developed splenomegaly, presumably the result of passive portal hypertension, and so an alternate diagnosis to bacterial endocarditis was never considered throughout the long course of the illness.

Even if such obvious cardiac signs are lacking, recurrent pulmonary emboli can cause a sustained fever, and should always be considered in the differential diagnosis of pyrexia.

Jaundice occurring in patients with heart failure frequently follows pulmonary infarction (Keefer and Resnick, 1926), whereas smaller infarcts occurring in individuals not in failure do not result in jaundice (Kugel and Lichtman, 1933). This is considered to be due to inability of the liver, damaged by cardiac failure, to excrete bile pigments liberated from infarcted tissues (Sherlock, I955). The presence of jaundice, ascites, hepatomegaly and melæna in Case 4 suggested a neoplastic lesion with hepatic metastases. In Case 3 pulmonary embolic disease, in addition to an associated neoplastic lesion, was considered likely, but in Case 4 the diagnosis was not considered at all, the likelihood of a primary gastro-intesinal neoplasm with hepatic secondaries seemed the most likely diagnosis.

Cerebro-vascular Disease. In all these cases the correct diagnosis was never considered. The features of cerebral tumour in Case 5 and cerebrovascular disease in Cases 6 and 7 seemed most likely. The development of fits and also of varying localized pyramidal signs was probably due to decreased cardiac output resulting in cerebrovascular insufficiency. At autopsy these cases had evidence of cerebral vascular disease, without demonstrable intra- or extra-cerebral arterial occlusion; and all during life were in cardiac failure with cyanosis. It is accepted that in such individuals anoxia can cause all the features which we have described and presumably in our case the sudden advent of coma, convulsions and hemiparesis followed the lodgement of further pulmonary emboli causing anoxia either by reduction of pulmonary gaseous exchange, or by a sudden fall in cardiac output. In view of the sudden onset of symptoms and their rapid subsidence it is probable that these features are due to a fall in cardiac output, comparable to the syncopal attacks which quite commonly herald the onset of this disease (Fleming, 1962).

As death occurred so soon after the onset of the 


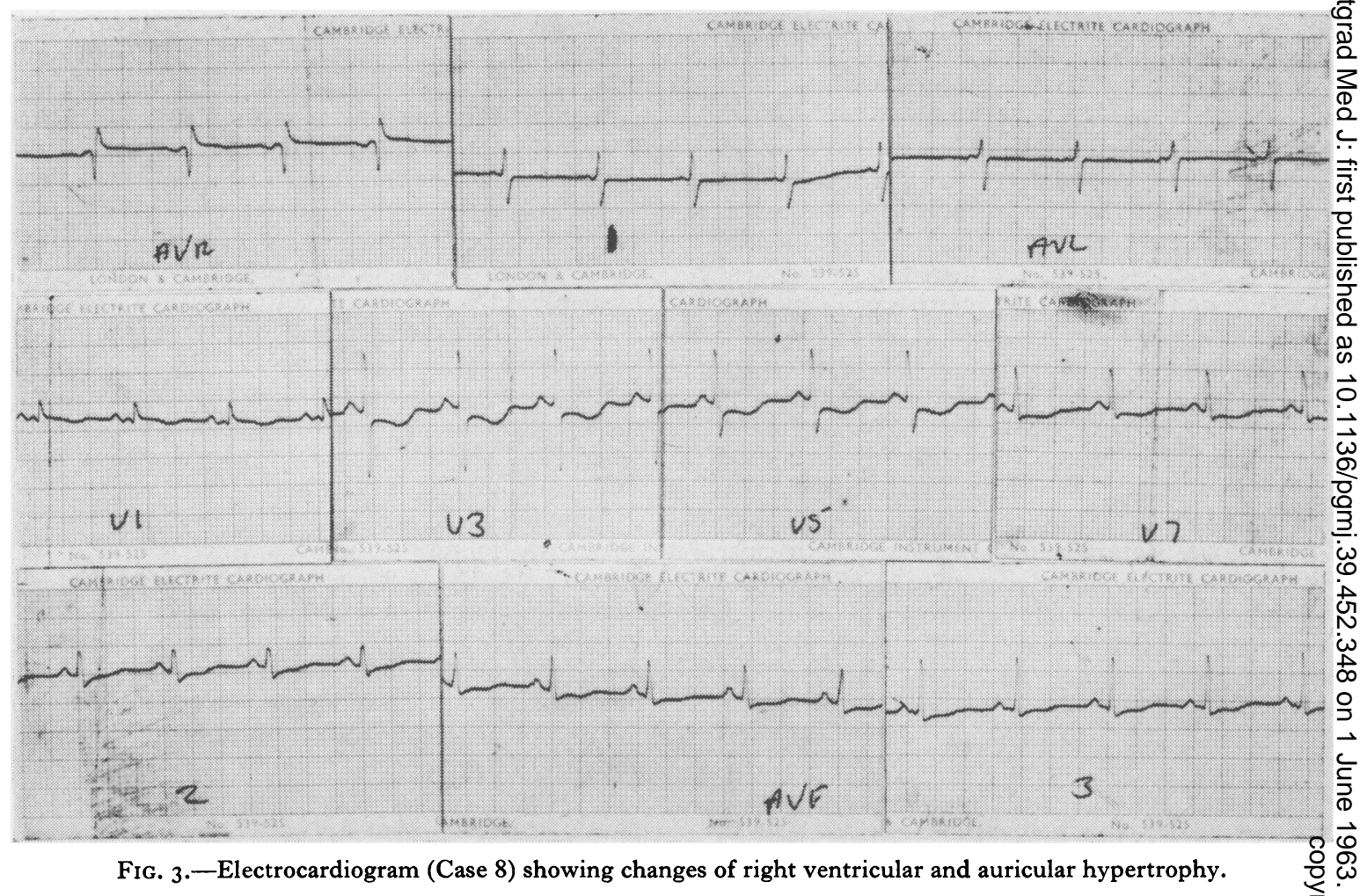

terminal convulsions, it seems likely that the decreased cardiac output secondary to terminal massive pulmonary embolus was the direct cause of the convulsions.

When this disease presents with neurological manifestations in a younger patient without evidence of arterial disease, the diagnosis becomes even more difficult, as illustrated by a further case recently under our care.

\section{Case 8}

A 49-year-old housewife admitted to hospital on 22.1 1.62 with a two-year history of increasing dyspnoea on effort. For the preceding II months she had experienced transient attacks of vertigo with ataxia and vomiting, each lasting for approximately one hour.

On admission to hospital she was in considerable heart failure, with the venous pressure raised 4 in. above the sternal angle, symmetrical œdema of both legs and râles at the lung bases. There was a definite right ventricular heave, with an ejection systolic murmur down the left sternal edge and accentuation of pulmonary valve closure, regular rhythm. B.P. $120 / 80 \mathrm{~mm}$. Hg. There was a striking trunk ataxia, but no other abnormality on neurological examination. There was no sign of leg vein thrombosis. The ECG (Fig. 3) showed changes of right ventricular and auricular hypertrophy; the chest $\mathrm{X}$-ray (Figs. 4 and 5 ) showed a considerable increase in the size of the heart and pulmonary arteries compared with that shown by an X-ray taken I I months previously, together with an area of consolidation in the right lower lobe.

Within 48 hours of admission to hospital, with no treatment except bed rest, all sign of cardiac failure haํํ엉 regressed. The remaining month of this patient's life was characterized by recurrent exacerbations of cardiac failure, each subsiding within 48 to 72 hours, and occurring despite maintenance treatment with digitalis $\mathbb{D}$ and diuretics, but never associated with chest pain or hæmoptysis. With each exacerbation the neurological $\overline{0}$ state deteriorated with progressive trunk ataxia, persistent 3 vomiting and the development and rapid progression of $\supset$ cerebellar dysarthria. The neurological picture was that of a rapidly extending lesion of the cerebellar vermis. For this reason a lumbar puncture was not performed, but an electroencephalograph report read that 'the EEG was of low voltage with a small bilateral alpha rhythm of normal frequency. A good deal of slow waves 3 . from the base line are present in the temporal regions. Apart from these doubtful abnormalities the EEG is normal. This supports the possibility of a posterior fossa tumour'.

The patient died suddenly one month after admission $>$ to hospital. Post-mortem examination showed that death was due to heart failure from widespread embolic obliteration of many small and large branches of both $N$ pulmonary arteries, with several areas of infarction in both lower lobes. There was extensive phlebothrombosis $N$ of the right leg veins. The basilar, carotid and vertebral $\underset{\mathrm{N}}{\mathbb{N}}$ arteries were normal; the brain showed odema, but no
other abnormality.

The great interest of this case lies in the development of focal neurological symptoms and? signs in a relatively young woman who, even at ${ }^{\circ}$ autopsy, showed no appreciable arterial disease. $\frac{\mathbb{D}}{\mathbb{D}}$ In retrospect, each exacerbation of cardiac failure 


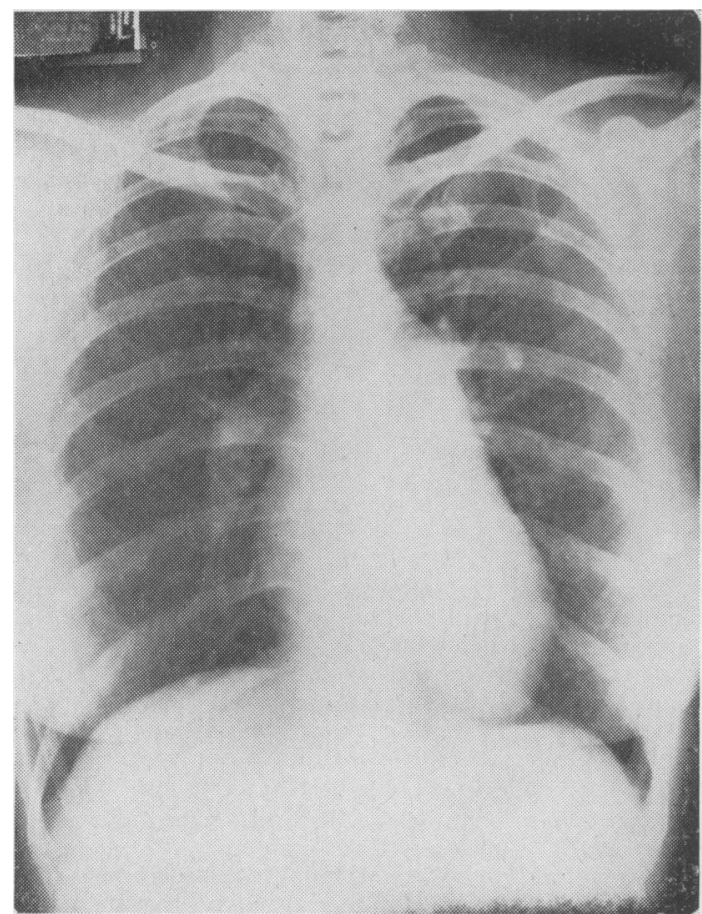

FIG. 4.-Chest X-ray (Case 8) taken I I months before admission to hospital.

was associated with a further deterioration in the neurological condition obviously both following further embolization to the lungs. However, during the patient's lifetime, although this association was apparent, its significance, now all too obvious, was ignored because the neurological condition was that of a rapidly expanding cerebellar lesion.

All these patients had been ambulant at the presumed onset of the pulmonary embolic disease, although latterly all were immobilized in bed by virtue of the terminal illness. The autopsy appearances of the lungs suggested long-standing pulmonary emboli in addition to the more recent massive terminal embolus. In some there was a

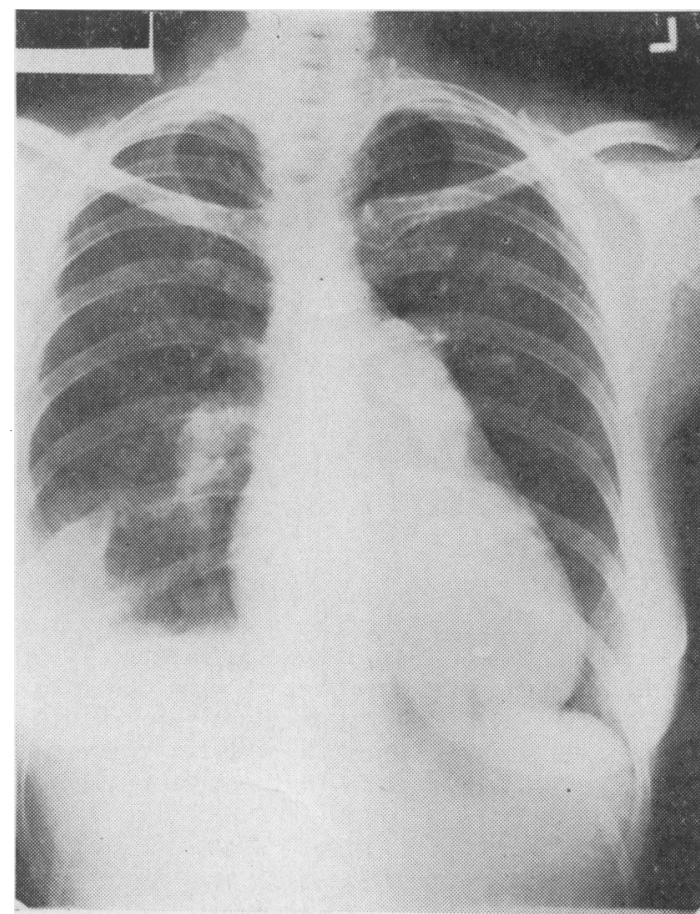

FIG. 5.-Chest X-ray (Case 8) showing classical changes of pulmonary embolic disease with cardiac enlargement, large hilar shadows and wedge-shaped peripheral consolidation.

previous history of phlebitis and also pulmonary emboli.

\section{Summary}

(I) Cases are described to illustrate unusual methods of presentation of pulmonary embolic disease: a condition which is becoming recognized with increasing frequency.

(2) Great difficulty was experienced in establishing the correct diagnosis as the presenting features of the disease closely resembled, bacterial endocarditis, cerebrovascular disease and gastrointestinal hæmorrhage.

(3) The mechanism by which such manifestations arose is discussed.

\section{REFERENCES}

Fleming, H. A. (1962): Pulmonary Embolic Disease, Lancet, ii, 45.

KeEFer, C. S., and Resnick, W. H. (1926): Jaundice following Pulmonary Infarction in Patients with Myocardial Insufficiency, $\boldsymbol{F}$. clin. Invest., 2, 375 .

Kugel, M. A., and Lichtman, S. S. (1933): Factors Causing Clinical Jaundice in Heart Disease, Arch. intern. Med., 52, 16.

Owen, W. R., Thomas, W. A., Castelman, B., and Bland, E. (I953): Unrecognised Emboli to Lungs with subsequent Cor Pulmonale, New Engl. F. Med., 249, 919.

SHERLOCK, S. (1955): 'Diseases of Liver and Biliary System', p. 406. Oxford: Blackwell.

Thompson, E. N., and Hamilton, M. (1962): Pulmonary Embolic Disease, Lancet, i, I369. 